\title{
CAPACIDAD BIOCONTROLADORA DE AISLAMIENTOS NATIVOS DE Trichoderma sp. CONTRA EL HONGO FITOPATÓGENO Alternaria alternata, AISLADO DE YERBA MATE (Ilex paraguariensis Saint Hil.)
}

\author{
BIOCONTROL CAPACITY OF NATIVE ISOLATES OF Trichoderma sp. \\ AGAINST THE PHYTOPATHOGENIC FUNGUS Alternaria alternata \\ ISOLATED FROM YERBA MATE (Ilex paraguariensis Saint Hil.)
}

\author{
María Castrillo $^{1 \mathrm{a}, 2 *}$, Gustavo Bich ${ }^{1 \mathrm{~b}, 2}$, Gastón Siolii ${ }^{1 \mathrm{c}}$, Pedro Zapata ${ }^{1 \mathrm{~d}, 2}$ y Laura Villalba ${ }^{1 \mathrm{e}}$
}

1a Universidad Nacional de Misiones, Instituto de Biotecnología Misiones "Dra. María Ebe Reca", Laboratorio de Biotecnología Molecular. Posadas, Argentina

https://orcid.org/0000-0002-4999-1672

1b Universidad Nacional de Misiones, Instituto de Biotecnología Misiones "Dra. María Ebe Reca", Laboratorio de Biotecnología Molecular. Posadas, Argentina https://orcid.org/0000-0002-3658-5157

1c Universidad Nacional de Misiones, Instituto de Biotecnología Misiones "Dra. María Ebe Reca", Laboratorio de Biotecnología Molecular. Posadas, Argentina https://orcid.org/0000-0003-1766-4658

1d Universidad Nacional de Misiones, Instituto de Biotecnología Misiones "Dra. María Ebe Reca", Laboratorio de Biotecnología Molecular. Posadas, Argentina

https://orcid.org/0000-0001-6476-8324

1e Universidad Nacional de Misiones, Instituto de Biotecnología Misiones "Dra. María Ebe Reca", Laboratorio de Biotecnología Molecular. Posadas, Argentina https://orcid.org/0000-0002-7488-4238

2 CONICET (Consejo Nacional de Investigaciones Científicas y Técnicas), Buenos Aires, Argentina

* Autor para correspondencia. mlc_827@hotmail.com

\section{RESUMEN}

Los hongos fitopatógenos afectan a cultivos agrícolas, como la yerba mate (Ilex paraguariensis Saint Hil.). Una alternativa innovadora para el control de plagas es el empleo de hongos biocontroladores del género Trichoderma. Los objetivos del presente trabajo fueron aislar los agentes fúngicos causantes de lesiones foliares en yerba mate, y evaluar la capacidad antagónica de los aislamientos nativos de Trichoderma sp. frente a patógenos en este cultivo. A partir del corte, desinfección y siembra del tejido vegetal enfermo, se aisló e identificó morfológica y molecularmente un hongo fitopatógeno perteneciente a la especie Alternaria alternata. Se ensayaron pruebas de patogenicidad en plantas de yerba mate por triplicado. Se realizaron lesiones mecánicas superficiales en ellas y se inoculó el aislamiento fitopatógeno obtenido (conidios y micelio) para evaluar su capacidad fitopatógena. Se determinaron nuevas variantes de cultivo dual para evaluar la capacidad antagónica biocontroladora en condiciones in vitro. Se evaluó la capacidad biocontroladora in vitro de 30 aislamientos de Trichoderma frente al aislamiento fitopatógeno obtenido. Como control positivo se utilizó una cepa aislada de un producto fungicida comercial a base de T. harzianum. Se determinó que 18 de los 30 aislamientos de Trichoderma lograron inhibir en más del 50\% el crecimiento del fitopatógeno A. alternata y presentaron diferencias estadísticamente significativas con respecto al 
control positivo. Estos resultados reafirman la efectividad de utilizar aislamientos biocontroladores nativos ya adaptados a las condiciones edafo-climáticas del ambiente en donde se encuentra el microorganismo fitopatógeno a controlar biológicamente.

Palabras clave: biocontrol, lesiones foliares, hongos antagonistas, cultivo dual, micoparasitismo.

\section{ABSTRACT}

Phytopathogenic fungi attack many agricultural crops such as yerba mate (Ilex paraguariensis Saint Hil.). An innovative alternative for pest control is the use of biocontrol fungi of Trichoderma genus. The objectives of the present work were to isolate the fungal agents that cause foliar lesions in yerba mate, and to evaluate the antagonistic capacity of native Trichoderma sp. isolates against pathogens in this crop. From diseased plant-tissues, a phytopathogenic fungus belonging to Alternaria alternata species was isolated and identified morphologically and molecularly. Pathogenicity assays were done on yerba mate plants in triplicate. Superficial mechanical lesions were made on them and the phytopathogenic isolation obtained (conidia and mycelium) was inoculated to evaluate their phytopathogenic capacity. New dual culture methods were determined to evaluate in vitro antagonism of Trichoderma. The in vitro biocontrol capacity of 30 Trichoderma isolates was evaluated against the phytopathogenic isolation obtained. As a positive control, a strain isolated from a commercial fungicide product based on $T$. harzianum was used. It was determined that 18 of the 30 isolates of Trichoderma inhibited the growth of the phytopathogen A. alternata by more than $50 \%$, and presented statistically significant differences with respect to the positive control. These results confirm the effectiveness of using native biocontrol isolates adapted to the climatic and soil conditions where the phytopathogenic microorganisms are found.

Key words: biocontrol, foliar lesions, antagonist fungi, dual culture, mycoparasitism.

\section{INTRODUCCIÓN}

La yerba mate (Ilex paraguariensis Saint Hil.) es una planta que crece en los países sudamericanos de Argentina, Brasil, Chile, Paraguay y Uruguay (Burris et al., 2012). Esta especie se encuentra naturalmente en suelos lateríticos ("tierra colorada") como parte del estrato del sotobosque (Alonso y Desmarchelier 2005; Laczeski et al., 2020). Los principales países productores y elaboradores de la materia prima son Paraguay $(4 \%)$, Brasil $(34 \%)$ y Argentina (62\%) (Debat et al., 2014). En Argentina, el relevamiento aéreo realizado por el Instituto Nacional de la Yerba Mate (INYM) en 2016 determinó que las zonas cultivadas con yerba mate representan una superficie de 165.200 ha, de las cuales 144.014 ha se encuentran en la provincia de Misiones y el resto en Corrientes (INYM, 2019; Laczeski et al., 2020).

A medida que se expandió el cultivo en la provincia de Misiones, Argentina, también se incrementaron las poblaciones de plagas. La activa participación del productor con el fin de lograr mejores y mayores rindes, contribuyó al mismo tiempo a perturbar la comunidad naturalmente equilibrada, por lo que se requiere una cantidad significativa de productos químicos para su cultivo y producción (Debat et al., 2014; Laczeski et al., 2020; Croge et al., 2021).

En la actualidad se ha profundizado en la investigación y posible utilización de fungicidas compuestos por antagonistas microbianos y productos vegetales que sean menos perjudiciales para el medio ambiente (Da Silva et al., 2017). Una alternativa de biocontrol de plagas se corresponde al uso de formulados con Trichoderma sp. (Hernández-Melchor et al., 2019; Ferreira et al., 2020; Moya et al., 2020).

El género Trichoderma (Persoon, 1794) incluye a especies fúngicas de vida libre en suelos y ecosistemas de raíz (Harman et al., 2004; Druzhinina, 2011). Presentan una distribución cosmopolita, bajo requerimiento nutrimental, crecimiento rápido y amplia plasticidad ecológica (Infante et al., 2009; Pérez et al., 2016). Este género posee una gran importancia desde el punto de vista económico, ambiental y biotecnológico. Es utilizado como agente de biocontrol contra hongos fitopatógenos, debido a sus múltiples mecanismos de acción, destacando la antibiosis, el micoparasitismo, la competencia por espacio y nutrientes, y la producción de metabolitos secundarios (Benítez et al., 2004; HernándezMelchor et al., 2019; Ferreira et al., 2020).

La evaluación de diferentes aislamientos nativos de Trichoderma sp. frente a diversos fitopatógenos de $I$. paraguariensis es una alternativa importante que podría contribuir a la utilización de diferentes estrategias de biocontrol en enfermedades que atacan al cultivo. Por lo tanto, el objetivo general fue aislar posibles 
agentes fúngicos causantes de lesiones foliares en I. paraguariensis y evaluar la capacidad antagónica de los aislamientos nativos de Trichoderma sp. frente a fitopatógenos.

\section{MATERIALES Y MÉTODOS}

\section{Material vegetal}

Con el propósito de identificar los agentes fúngicos que causan lesiones foliares en diferentes plantas de yerba mate (I. paraguariensis Saint Hil.), se examinaron hojas y tallos con signos o lesiones sospechosas de ser causadas por hongos fitopatógenos. Las muestras fueron colectadas en el vivero orgánico de la Fundación Alberto Roth

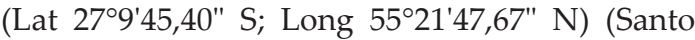
Pipó, Misiones, Argentina) en diferentes épocas del año. A cada una de las muestras colectadas se las etiquetó y colocó en bolsas de papel de primer uso para ser transportadas al laboratorio.

\section{Aislamiento e identificación de hongos fitopatógenos de yerba mate}

Para el aislamiento del posible agente fitopatógeno, se procedió a realizar cortes del material biológico vegetal enfermo en porciones cuadradas de aproximadamente $5 \mathrm{~mm}$ de diámetro, desde el borde de la lesión. Estos cortes fueron desinfectados externamente, se sumergieron $1 \mathrm{~min}$ en hipoclorito de sodio al $1 \%$, y se enjuagaron en alcohol $70 \%$ y dos veces en agua destilada estéril por 2 min con agitación. Se tomaron porciones del material vegetal desinfectado y se sembraron en placas con agar-agua al 2\% adicionadas con el antibiótico cloranfenicol a una concentración de 100mg/L (Salvatierra-Martínez et al., 2015). Las placas se incubaron a $28 \pm 1{ }^{\circ} \mathrm{C}$ hasta la visualización de colonias fúngicas (aproximadamente 7 días) bajo condiciones de fotoperiodo controlado (12 h luz: $12 \mathrm{~h}$ oscuridad).

Luego del periodo de incubación se observó el desarrollo de varias colonias fúngicas (de 2 a 4 colonias fúngicas por placa) con similares características morfológicas. Se identificó el aislamiento perteneciente a un género fúngico fitopatógeno de acuerdo con las características morfológicas macroscópicas y microscópicas (Barnett and Hunter, 1998; Perelló et al., 2015; Piontelli Laforet, 2017). Este aislamiento se repicó hasta obtener un cultivo puro y se conservó a $4^{\circ} \mathrm{C}$.

Con la finalidad de identificar molecularmente al aislamiento fitopatógeno se procedió a extraer ADN empleando los protocolos estandarizados por Hermosa et al. (2000) y Bich et al. (2017). Se amplificó la región ITS1-5.8S-ITS2 utilizando los cebadores universales ITS1 e ITS4 (White et al., 1990; Schoch et al., 2012), en un volumen de reacción de $20 \mu \mathrm{L}$ (Castrillo et al., 2016). Los productos de amplificación fueron evaluados en geles de agarosa al 2\%, y luego secuenciados utilizando el servicio de Macrogen Korea. Posteriormente, fueron analizados con el software bioinformático Geneious 8 que permite la visualización de los cromatogramas, facilita su control y la conformación de una secuencia cóntigo consenso. Estas secuencias consenso fueron contrastadas con las bases de datos genéticas del Fungal Barcoding (https://wi.knaw. nl/page/Pairwise_alignment) y del National Center for Biotechnology Information (NCBI) (http://blast.ncbi.nlm.nih.gov).

\section{Patogenicidad del microorganismo fitopatógeno}

Asimismo, con la finalidad de verificar si el aislamiento fitopatógeno obtenido desarrollaba sintomatología similar a la observada en la planta afectada, se realizaron pruebas de patogenicidad (Hosainzadegan et al., 2020). En este caso se desinfectaron externamente hojas y tallos de plantines de yerba mate orgánicos de $25 \mathrm{~cm}$ de altura, y se realizaron lesiones mecánicas superficiales en estos tejidos vegetales, con la ayuda de un bisturí estéril. Se tomaron porciones de cultivos en placa del aislamiento fitopatógeno obtenido (conidios y micelio), mediante el empleo de un ansa estéril se infectaron por contacto directo las lesiones de las plantas de yerba mate, y restringieron estas lesiones del ambiente con plástico transparente. Estos plantines fueron conservados en condiciones controladas de humedad ambiental $\geq 80 \%$ HR y luz (12 h luz: 12 h oscuridad) para mantener vivos a los plantines y registrar la posible infección de estos por el aislamiento fúngico inoculado. Como testigo se realizaron lesiones con un bisturí estéril en plantines de yerba mate orgánicos de igual edad de desarrollo. Estos plantines también se incubaron en similares condiciones que el tratamiento con el posible hongo fitopatógeno. Todos estos ensayos fueron realizados por triplicado.

\section{Cepas de Trichoderma}

Como hongos antagonistas del aislamiento fitopatógeno se utilizaron 30 aislamientos diferentes del género Trichoderma, pertenecientes al cepario de interés biotecnológico de la Universidad Nacional de Misiones, Argentina. Los aislamientos consistieron en 29 cepas aisladas de ecosistemas naturales de la provincia de Misiones, Argentina (T. koningiopsis NAN9, T. koningiopsis NAN11, T. pleroticola NAN12, T. harzianum NAN13, T. harzianum POS1, T. atroviride POS2, T. harzianum POS3, T. longibrachiatum POS5, T. longibrachiatum POS6, T. koningiopsis POS7, T. atroviride POS8, T. reesei POS9, T. 
longibrachiatum POS10, T. stilbohypoxyli POS11, T. brevicompactum PROF1, T. harzianum PROF2, T. harzianum PROF3, T. koningiopsis PROF4, T. koningiopsis PROF5, T. harzianum PROF6, T. harzianum PROF7, T. koningiopsis PROF8, T. harzianum TEYU14, T. longibrachiatum TN1, T. asperellum $\mathrm{TN} 2, \mathrm{~T}$. harzianum $\mathrm{TN} 3, \mathrm{~T}$. harzianum TN4, T. citrinoviride TN5, T. koningiopsis TrichoH) y una cepa aislada de un producto fungicida comercial a base de T. harzianum (TrichoX, Yumbo, Colombia).

Capacidad biocontroladora in vitro de aislamientos de Trichoderma contra el hongo fitopatógeno aislado

Se evaluaron tres métodos diferentes de cultivo dual para la determinación de la capacidad biocontroladora antagónica en placas de Petri de $90 \mathrm{~mm}$ de diámetro. Como base, se siguió la metodología descrita por Ab Rahman et al. (2018) y Guo et al. (2019), la cual consiste en colocar en puntos opuestos de una placa de Petri, conteniendo agar papa dextrosa (PDA) (Britania Lab, Buenos Aires, Argentina) como medio de cultivo, un disco de $7 \mathrm{~mm}$ de diámetro de agar con micelio esporulado del fitopatógeno, y en el otro extremo, un disco de agar con micelio esporulado de la cepa de Trichoderma. Además, de la forma tradicional descripta, se evaluaron dos nuevas variantes del método de cultivo dual: una, donde se colocó un disco con micelio esporulado de Trichoderma en cada extremo y un solo disco con micelio esporulado del fitopatógeno en el centro de la placa; y otra, donde se dispuso un disco con micelio esporulado de Trichoderma en el centro y un disco con micelio del fitopatógeno en cada extremo de la placa (Fig. 1).

Para determinar cuál de estos tres métodos de cultivo dual ensayados fue más eficiente, se realizó un análisis de varianza (ANOVA) utilizando el software STATGRAPHICS Centurion XV.II para Windows.

Como control experimental, se utilizó la cepa proveniente del producto fungicida comercial (TrichoX). Cada ensayo se realizó por triplicado y se obtuvieron registros diarios del radio de crecimiento de cada aislamiento por un período de 10 días a $28 \pm 1^{\circ} \mathrm{C}$.

Además, se cultivaron individualmente el aislamiento fitopatógeno obtenido y cada uno de los 30 aislamientos del género Trichoderma a fin de observar su desarrollo y obtener las mediciones correspondientes a su crecimiento. Este registro se realizó por un período de 10 días de incubación a $28 \pm 1^{\circ} \mathrm{C}$.

La eficacia del antagonista en inhibir el crecimiento del fitopatógeno fue evaluada cuantitativamente mediante la fórmula de Abbott adaptada (Andrade-Hoyos et al., 2020; Choudhary et al., 2021).

$$
G I=(A p-A p+a) x 100 / A p
$$

donde Ap es el crecimiento radial del patógeno y Ap+a es el crecimiento del patógeno en presencia del antagonista, y GI es el grado de inhibición en porcentaje.

Asimismo, se empleó una escala que evalúa cualitativamente la capacidad antagónica que posee cada aislamiento de Trichoderma en invadir y reducir la colonia del hongo fitopatógeno (Bell et al., 1982; Andrade-Hoyos et al., 2020).

Para evaluar el modo de acción micoparasítico de Trichoderma, se realizaron preparaciones microscópicas para observar estrategias tales como la penetración y el enrollamiento del micelio del patógeno. Se realizaron preparaciones microscópicas de las zonas de contacto entre Trichoderma y el hongo fitopatógeno y se

\section{Enfrentamiento por cultivo dual}
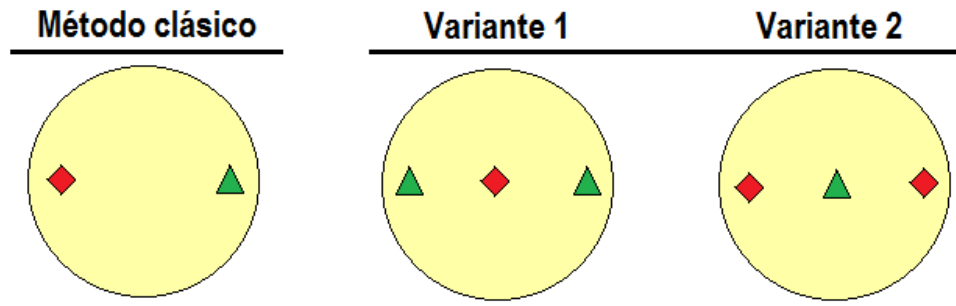

\section{Fitopatógeno}

$\triangle$ Trichoderma

Fig. 1. Diferentes ensayos de cultivo dual para evaluar la capacidad biocontroladora antagónica de Trichoderma sp.

Fig. 1. Different dual culture assays to evaluate the antagonistic biocontrol capacity of Trichoderma sp. 
observaron bajo el microscopio óptico Carl Zeiss - model 467065-9902-18VA, con aumentos de 10X a 100X (Burgess and Hepworth, 1996).

\section{RESULTADOS Y DISCUSIÓN}

Aislamiento e identificación de hongos fitopatógenos de plantines de yerba mate

En el proceso de domesticación delayerba mate, los efectos de las técnicas culturales (densidad, labores sanitarias, fertilización) y la erosión genética de las características de resistencia favorecen el desarrollo de enfermedades (Auer and Grigoletti, 2002; Laczeski et al., 2020; Croge et al., 2021). En estas circunstancias, los plantines jóvenes, plantines poco vigorosos o estresados, son más susceptibles y dan lugar a plantas más propensas a contraer enfermedades producidas por hongos fitopatógenos.

En campo se observó que las plantas de yerba mate presentaron signos de ataque por hongos fitopatógenos representadas por áreas de color marrón amarillento en los lóbulos de las hojas, que en partes se extendían desde la lámina foliar hasta el pecíolo y el tallo (Fig. 2 a). A partir del tercer día de incubación se comenzó a observar el desarrollo de dos a cuatro colonias fúngicas por placa con similares características morfológicas, lo que indicaría que las colonias fúngicas podían pertenecer a la misma especie. Estas colonias del potencial aislamiento fitopatógeno presentaron una coloración blanquecina con bordes regulares que se tornó pardo-grisácea con el mayor tiempo de cultivo. El reverso de las colonias presentó una coloración oscura. Microscópicamente se observaron conidióforos rectos o flexuosos, de coloración parda, septados y de longitud variable. En general, los conidios fueron piriformes, pardos, con septos longitudinales y transversales. Estas características macro y micromorfológicas particulares del aislamiento fúngico al séptimo día de incubación permitieron identificarlo como Alternaria alternata (Fig. 2 b).

La secuenciación de los fragmentos obtenidos, a través del uso de los cebadores universales empleados, permitió la obtención de secuencias de nucleótidos de buena calidad para los amplicones/productos de amplificación obtenidos del aislamiento fitopatógeno (Schoch et al., 2012). A fin de comparar, caracterizar e identificar las secuencias obtenidas con aquellas depositadas en las bases de datos, la secuencia cóntigo consenso generada fue contrastada mediante el motor de búsqueda de identidad y similitud de las bases de datos NCBI y Fungal barcoding. En las dos bases de datos se corroboró que el aislamiento fitopatógeno se identificó como perteneciente a la especie $A$. alternata, con índices de identidad máxima del $97,7 \%$ y $100 \%$ (KY026586 y DAOM 216376, según el motor de búsqueda del Fungal barcoding y NCBI, respectivamente). Esta secuencia cóntigo consenso fue depositada en la base de datos del NCBI bajo el número de acceso MG831329, y el aislamiento fue depositado
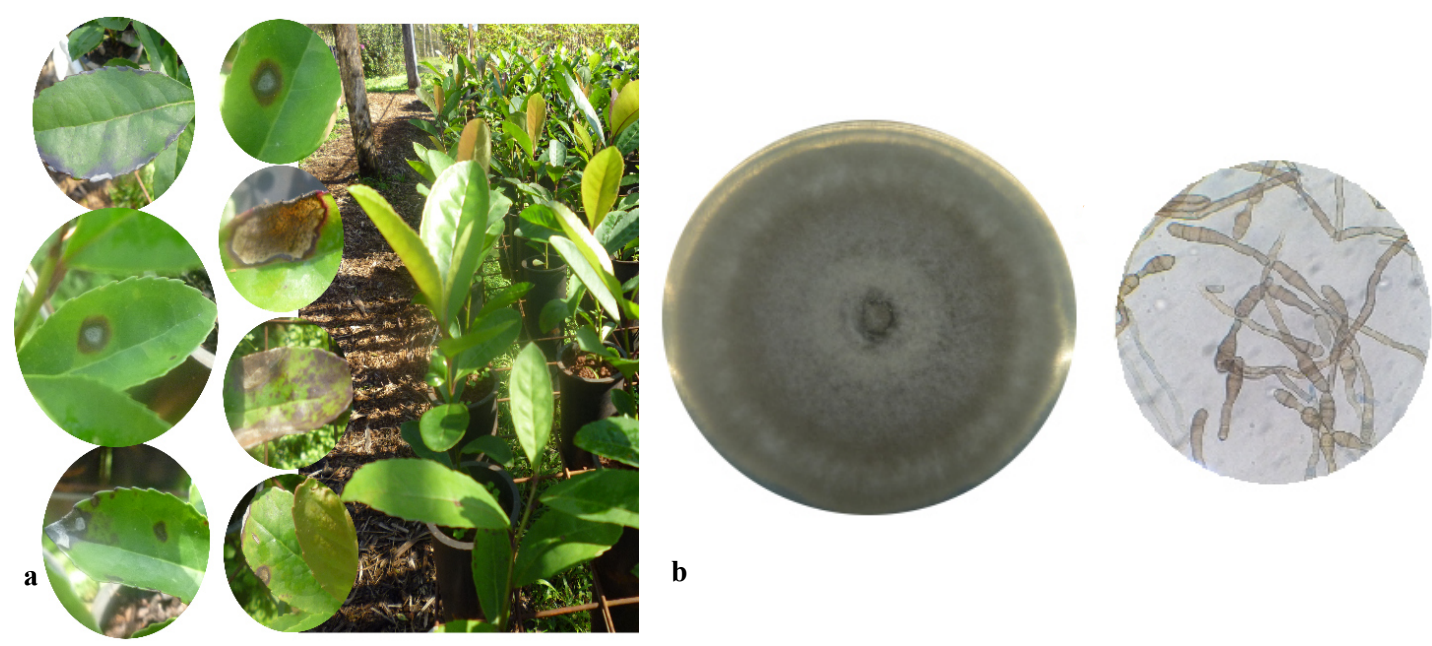

Fig. 2. a) Muestras de yerba mate (I. paraguariensis Saint Hil.) con signos de ataque por hongos fitopatógenos. b) Características macro y microscópicas observadas durante el crecimiento in vitro del hongo fitopatógeno A. alternata, en el medio de cultivo PDA.

Fig. 2. a) Yerba mate (I. paraguariensis Saint Hil.) samples with signs of attack by phytopathogenic fungi. b) Macro and microscopic features observed during the in vitro growth of the phytopathogenic fungus $A$. alternata in PDA culture medium. 
en el cepario de interés biotecnológico de la Universidad Nacional de Misiones bajo el código LBM124.

La caracterización morfológica del aislamiento fitopatógeno en el presente trabajo fue similar a los resultados obtenidos por Andersen et al. (2001), Cúndom y Cabrera (2002), Lezcano et al. (2009), Gauna et al. (2020), Abbas et al. (2021) y Aloi et al. (2021), para la especie A. alternata.

Numerosos autores han citado al género Alternaria como uno de los hongos fitopatógenos que causan mayores daños al follaje, a la germinación de las semillas y al desarrollo de los plantas de diversos vegetales (Fraire-Cordero et al., 2010; Perelló et al., 2015; Salvatierra-Martinez et al., 2015; Sadañoski et al., 2018; Gauna et al., 2020; Abbas et al., 2021), incluso, como agente causante de manchas foliares o marchitamiento vascular en I. paraguariensis (Auer y Grigoletti, 2002; Burtnik, 2006; Pérez et al., 2016; Fontana et al., 2021).

Confirmación de la patogenicidad del microorganismo fitopatógeno obtenido

Las pruebas de patogenicidad realizadas sobre plantines orgánicos de yerba mate permitieron corroborar que el aislamiento fitopatógeno obtenido posee la capacidad de desarrollar una sintomatología similar a la observada inicialmente en las plantas de yerba mate muestreadas. Estos resultados indican que el aislamiento fúngico aplicado fue capaz de infectar a los tejidos foliares y tallo de los plantines de yerba mate inoculados. Se observó una necrosis del tejido vegetal infectado y desarrollo de micelio del microorganismo aplicado (Fig. 3 a). Este micelio fue recuperado y observado nuevamente al microscopio óptico para corroborar que haya sido el microorganismo aplicado (Fig. $3 \mathrm{~b}$ ). Se observaron estructuras similares a las del aislamiento fúngico obtenido originalmente a partir de las muestras de yerba mate, por lo que se pudo confirmar que el hongo A. alternata es capaz de infectar plantines de yerba mate.

Capacidad biocontroladora in vitro de aislamientos de Trichoderma sp. contra el aislamiento fitopatógeno obtenido

Uno de los primeros pasos en la aplicación de agentes biocontroladores en programas de control de plagas es el correcto aislamiento e identificación del hongo fitopatógeno (Hermosa et al., 2000). Por lo tanto, una vez identificado y confirmado que $A$. alternata posee capacidad fitopatógena en plantas de yerba mate, se procedió a evaluar la capacidad biocontroladora de diferentes aislamientos del género Trichoderma, adaptados a las condiciones edafo-climáticas de la región, contra el aislamiento fitopatógeno obtenido.

Las especies pertenecientes al género Trichoderma suelen presentar éxito como agentes de control biológico debido a su capacidad reproductiva alta, crecimiento rápido, habilidad para sobrevivir bajo condiciones ambientales desfavorables, fuerte agresividad contra hongos
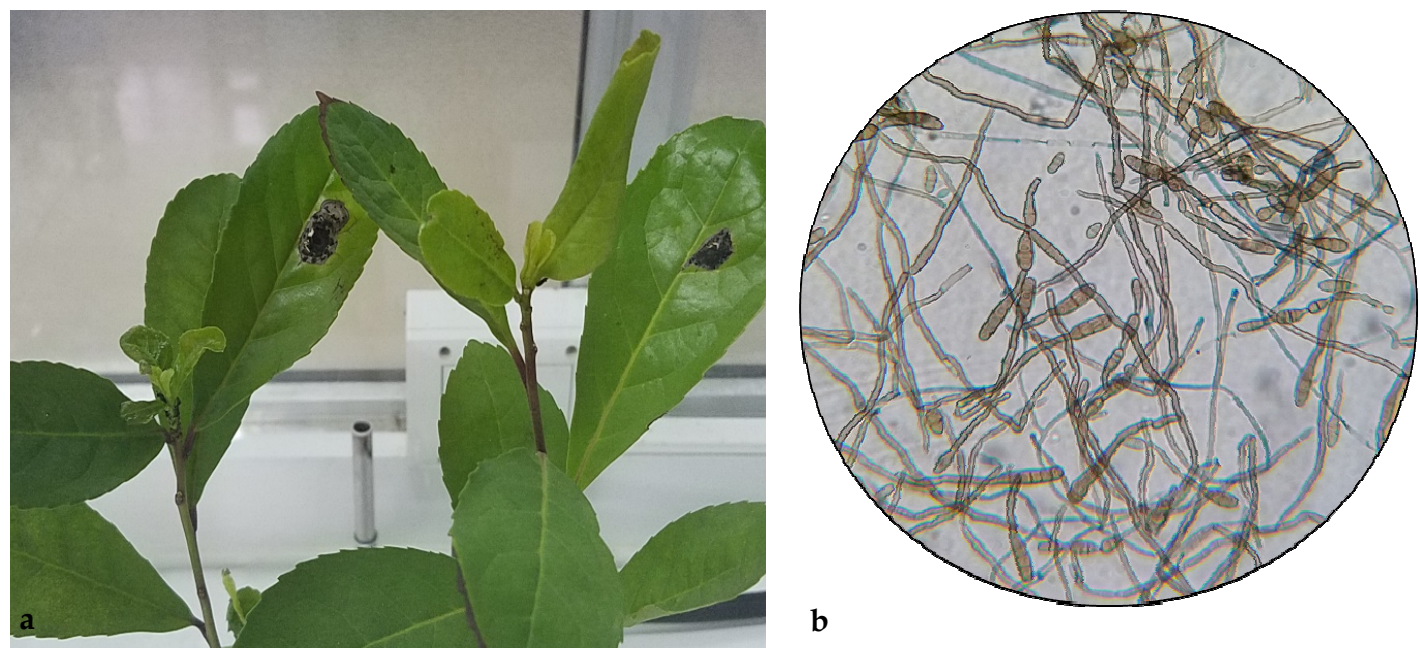

Fig. 3. a) Plantas de yerba mate (I. paraguariensis Saint Hil.) con signos de ataque fúngico por $A$. alternata. b) Características microscópicas observadas durante el crecimiento in vitro del hongo fitopatógeno re-aislado A. alternata, en el medio de cultivo PDA.

Fig. 3. a) Yerba mate (I. paraguariensis Saint Hil.) plants with signs of fungal attack by A. alternata. b) Microscopic characteristics observed during the in vitro growth of the phytopathogenic fungus A. alternata in PDA culture medium. 
fitopatógenos e inducción de mecanismos de defensa en vegetales (Bell et al., 1982; Benítez et al., 2004; Infante et al., 2009; Pérez et al., 2016; Hernández-Melchor et al., 2019).

El ANOVA realizado para determinar si existían diferencias significativas entre las tres variantes de cultivo dual, determinó que las variantes ensayadas no presentaron diferencias estadísticamente significativas ( $p>0,05)$, por lo que todas las variantes resultan útiles para verificar la capacidad antagónica de los aislamientos de Trichoderma. Sin embargo, en el presente trabajo se decidió optar por utilizar la variante 2, ya que esa condición podría arrojar resultados similares al contexto natural en los cuales se enfrentan estos microorganismos.

A partir de la selección de la variante de cultivo dual modificada fue posible evaluar la capacidad biocontroladora in vitro de todos los aislamientos de Trichoderma frente a A. alternata (Fig. 4). La eficacia de Trichoderma en inhibir el crecimiento del fitopatógeno fue evaluada cuantitativamente mediante la fórmula de Abbott adaptada (Andrade-Hoyos et al., 2020; Choudhary et al., 2021). Teniendo en cuenta que un buen aislamiento antagónico es aquel que logra inhibir en un 50\% o más el crecimiento colonial del fitopatógeno en un período de evaluación de 5 a 7 días de incubación, se observó que los aislamientos T. koningiopsis NAN11, T. pleroticola NAN12, T. harzianum POS1, T. atroviride POS2, T. longibrachiatum POS5, T. longibrachiatum POS6, T. koningiopsis POS7, T. longibrachiatum POS10, T. stilbohypoxyli POS11, T. harzianum PROF2, T. harzianum PROF3, T. koningiopsis PROF4, T. koningiopsis PROF5, T. harzianum PROF6, T. harzianum PROF7, T. koningiopsis PROF8, T. harzianum TEYU14, T. harzianum TN3, T. citrinoviride TN5 y T. koningiopsis TrichoH lograron inhibir significativamente el crecimiento del aislamiento fitopatógeno A. alternata (Tabla 1). Particularmente los aislamientos T. pleroticola NAN12, T. koningiopsis POS7, T. harzianum PROF3, T. koningiopsis PROF4, T. koningiopsis PROF5, T. harzianum PROF6 y T. harzianum TN3 mostraron una capacidad de inhibición mayor al $60 \%$ y presentaron diferencias estadísticamente significativas $(p<0,05)$ con respecto a los demás aislamientos evaluados y particularmente al ensayo realizado con la cepa obtenida del producto comercial (TrichoX).

De acuerdo a lo reportado por diversos autores (Andrade-Hoyos et al., 2020; Choudhary et al., 2021), se empleó la escala de Bell et al. (1982) para determinar cualitativamente la capacidad antagónica que posee cada uno de los 30 aislamientos de Trichoderma en invadir y reducir el crecimiento del fitopatógeno. Teniendo en cuenta que un índice 3 determina una capacidad antagónica satisfactoria para un antagonista (Calistru et al., 1997; Marraschi et al., 2019), se pudo determinar que los aislamientos $T$. koningiopsis NAN11, T. pleroticola NAN12, T. atroviride POS2, T. longibrachiatum POS5, T. longibrachiatum POS6, T. harzianum PROF2, T. harzianum PROF3, T. koningiopsis PROF4, T. harzianum PROF6, T. harzianum PROF7, T. koningiopsis PROF8, T. harzianum TEYU14, T. harzianum TN3 y $T$. harzianum TN4 presentaron un índice clase 3 y los aislamientos T. harzianum NAN13, T. harzianum POS1, T. koningiopsis POS7, T. atroviride POS8, T. stilbohypoxyli POS11, T. brevicompactum PROF1, T. koningiopsis PROF5 y T. koningiopsis TrichoH presentaron un índice clase 4 (Fig. 4).

Resultados similares a los reportados en el presente trabajo fueron obtenidos por Chaverri et al. (2015), Toghueo et al. (2016), Andrade-Hoyos et al. (2020), Ferreira et al. (2020) y Choudhary et al. (2021). Todos estos autores observaron que las diferencias en el \% GI variaba a nivel de cepa y no de especie, ya que diferentes cepas de una misma especie del género Trichoderma presentaba algunas cepas con gran potencial biocontrolador in vitro y otras cepas lo contrario.

En el presente trabajo se pudo observar que la mayoría de los aislamientos de Trichoderma evaluados produjeron una reducción estadísticamente significativa $(\mathrm{p}<0,05)$ en el crecimiento del hongo fitopatógeno A. alternata en el enfrentamiento de cultivo dual. Estos resultados pueden deberse a que, al tratarse de aislamientos de Trichoderma que se encuentran naturalmente en la misma zona que el hongo fitopatógeno aislado y los cultivos de yerba mate, se asegura que los aislamientos de Trichoderma estén adaptados al ecosistema y a las condiciones climáticas y edáficas locales, derivando en una interacción hongo antagonista hongo fitopatógeno suelo y ambiente más adecuada que la inoculación con productos comerciales a base de cepas de Trichoderma exóticas (Raza et al., 2019; Canassa et al., 2020; Laczeski et al., 2020). Ommati y Zaker (2012), Raza et al. (2019) y Canassa et al. (2020) reportaron que una estrategia útil para el uso de aislamientos de Trichoderma en el control biológico de plagas, es identificar especies que se encuentren adaptadas a los diferentes agroecosistemas de las plagas.

Particularmente, la cepa T. harzianum TrichoX utilizada como control experimental presentó una capacidad cuantitativa de inhibición del crecimiento del fitopatógeno cercana al 50\% y un índice de antagonismo de Bell et al. (1982) de clase 1. Esto brinda una prueba de la utilidad y las ventajas que se pueden obtener al utilizar los recursos disponibles regionalmente (Raza et al., 2019; Canassa et al., 2020; Laczeski et al., 2020), aprovechando la gran biodiversidad de la selva 

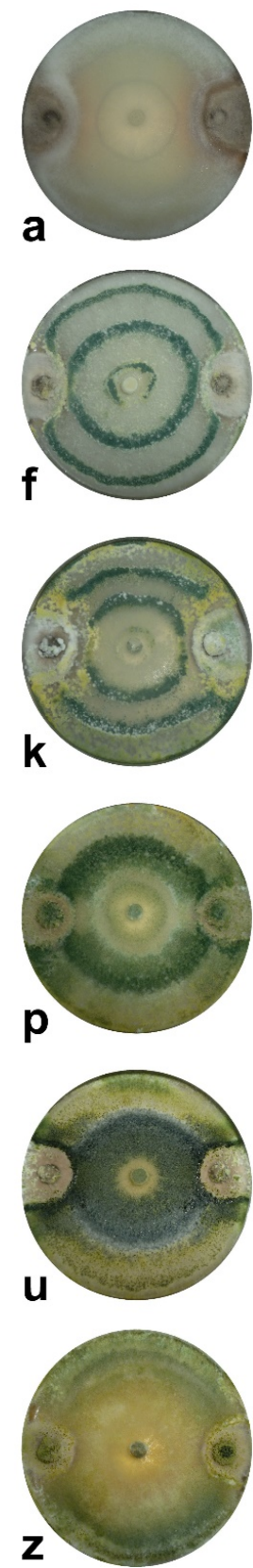
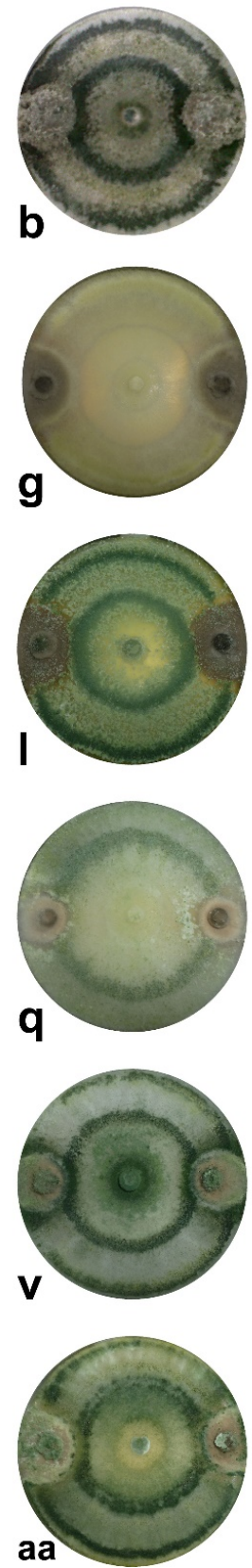
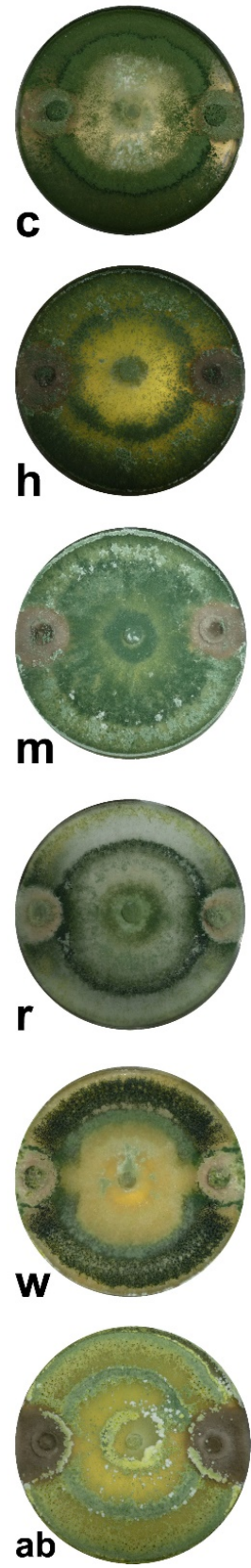
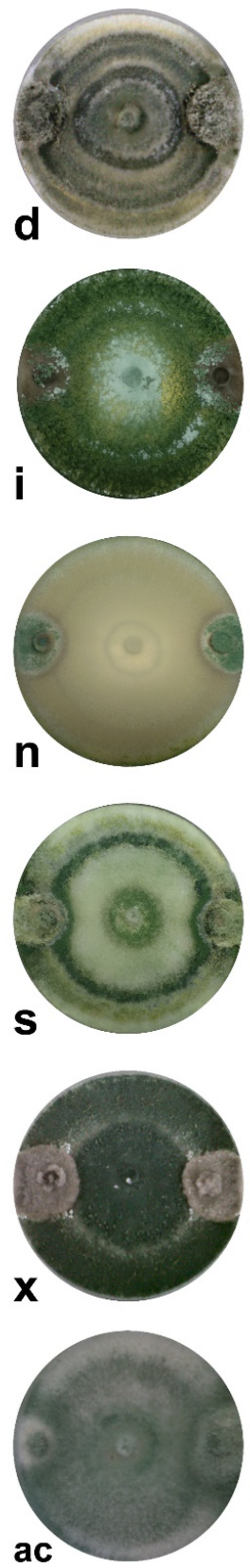
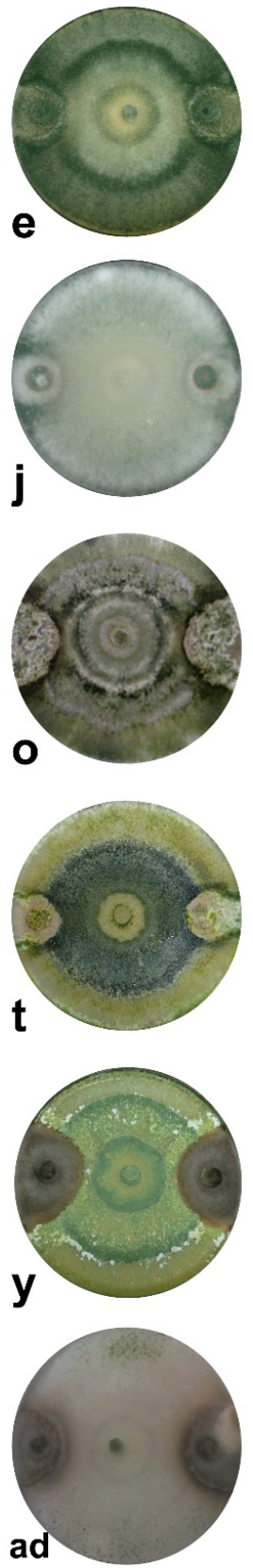

Fig. 4. Antagonismo en cultivo dual de los 30 aislamientos de Trichoderma sp. frente al hongo fitopatógeno A. alternata. a) T. koningiopsis NAN9. b) T. koningiopsis NAN11. c) T. pleroticola NAN12. d) T. harzianum NAN13. e) T. harzianum POS1. f) T. atroviride POS2. g) T. harzianum POS3 h) T. longibrachiatum POS5. i) T. longibrachiatum POS6. j) T. koningiopsis POS7. k) T. atroviride POS8. 1) T. reesei POS9. m) T. longibrachiatum POS10. n) T. stilbohypoxyli POS 11. o) T. brevicompactum PROF1. p) T. harzianum PROF2. q) T. harzianum PROF 3. r) T. koningiopsis PROF4. s) T. koningiopsis PROF5. t) T. harzianum PROF6. u) T. harzianum PROF7. v) T. koningiopsis PROF8. w) T. harzianum TEYU14. x) T. longibrachiatum TN1. y) T. asperellum TN2. z) T. harzianum TN3. aa) T. harzianum TN4. ab) T. citrinoviride TN5. ac) T. koningiopsis TrichoH. ad) T. harzianum TrichoX. En el centro de cada placa de Petri se encuentra Trichoderma y en los extremos opuestos $A$. alternata. Las fotografías corresponden al décimo día de incubación.

Fig. 4. Antagonism in dual culture of the 30 isolates of Trichoderma sp. against the phytopathogenic fungus A. alternata. a) T. koningiopsis NAN9. b) T. koningiopsis NAN11. c) T. pleroticola NAN12. d) T. harzianum NAN13. e) T. harzianum POS1. f) T. atroviride POS2. g) T. harzianum POS3 h) T. longibrachiatum POS5. i) T. longibrachiatum POS6. j) T. koningiopsis POS7. k) T. 
atroviride POS8. 1) T. reesei POS9. m) T. longibrachiatum POS10. n) T. stilbohypoxyli POS 11. o) T. brevicompactum PROF1. p) T. harzianum PROF2. q) T. harzianum PROF 3. r) T. koningiopsis PROF4. s) T. koningiopsis PROF5. t) T. harzianum PROF6. u) T. harzianum PROF7. v) T. koningiopsis PROF8. w) T. harzianum TEYU14. x) T. longibrachiatum TN1. y) T. asperellum TN2. z) T. harzianum TN3. aa) T. harzianum TN4. ab) T. citrinoviride TN5. ac) T. koningiopsis TrichoH. ad) T. harzianum TrichoX. Trichoderma is located in the center of each Petri dish and A. alternata at the opposite ends. Photographs taken on the tenth day of incubation.

Tabla 1. Grados de inhibición (GI) para cada uno de los 30 aislamientos de Trichoderma sp. frente al aislamiento fitopatógeno A. alternata.

Table 1. Degrees of inhibition (GI) for each of the 30 isolates of Trichoderma sp. versus the phytopathogenic isolate $A$. alternata.

\begin{tabular}{|c|c|c|c|}
\hline Antagonista & $\begin{array}{c}\text { GI \% } \\
\text { A. alternata }\end{array}$ & Antagonista & $\begin{array}{c}\text { GI \% } \\
\text { A. alternata }\end{array}$ \\
\hline T. koningiopsis NAN9 & $49,09 \pm 0,10^{b}$ & T. atroviride POS8 & $41,27 \pm 0,06^{a}$ \\
\hline T. koningiopsis NAN11 & $53,27 \pm 0,05^{c}$ & T. reesei POS9 & $46,36 \pm 0,11^{b}$ \\
\hline T. pleroticola NAN12 & $61,82 \pm 0,10^{d}$ & T. longibrachiatum POS10 & $58,73 \pm 0,30^{c}$ \\
\hline T. harzianum NAN13 & $46,91 \pm 0,20^{\mathrm{b}}$ & T. stilbohypoxyli POS11 & $58,18 \pm 0,10^{c}$ \\
\hline T. harzianum POS1 & $54,55 \pm 0,07^{c}$ & T. brevicompactum PROF1 & $41,09 \pm 0,15^{a}$ \\
\hline T. atroviride POS2 & $54,55 \pm 0,10^{c}$ & T. harzianum PROF2 & $56,36 \pm 0,08^{c}$ \\
\hline T. harzianum POS3 & $47,27 \pm 0,30^{\mathrm{b}}$ & T. harzianum PROF3 & $63,64 \pm 0,12^{d}$ \\
\hline T. longibrachiatum POS5 & $58,18 \pm 0,09^{c}$ & T. koningiopsis PROF4 & $60 \pm 0,18^{d}$ \\
\hline T. longibrachiatum POS6 & $56,36 \pm 0,02^{\mathrm{c}}$ & T. koningiopsis PROF5 & $62,73 \pm 0,07^{\mathrm{d}}$ \\
\hline T. koningiopsis POS7 & $63,09 \pm 0,04^{d}$ & T. harzianum PROF6 & $61,82 \pm 0,10^{d}$ \\
\hline T. harzianum PROF7 & $56,36 \pm 0,20^{c}$ & T. harzianum TN3 & $60 \pm 0,15^{\mathrm{d}}$ \\
\hline T. koningiopsis PROF8 & $58,18 \pm 0,09^{c}$ & T. harzianum TN4 & $48,55 \pm 0,07^{\mathrm{b}}$ \\
\hline T. harzianum TEYU14 & $58,18 \pm 0,10^{c}$ & T. citrinoviride TN5 & $50 \pm 0,03^{b, c}$ \\
\hline T. longibrachiatum TN1 & $47,64 \pm 0,05^{b}$ & T. koningiopsis TrichoH & $51,82 \pm 0,04^{b, c}$ \\
\hline T. asperellum TN2 & $37,27 \pm 0,13^{a}$ & T. harzianum TrichoX & $49,09 \pm 0,12^{b}$ \\
\hline
\end{tabular}

Nota: GI presenta la media y el desvío estándar de los ensayos por triplicado para cada aislamiento nativo biocontrolador de Trichoderma. Las letras indican las diferencias significativas entre los GI (prueba de LSD, $\mathrm{p}<0,05)$.

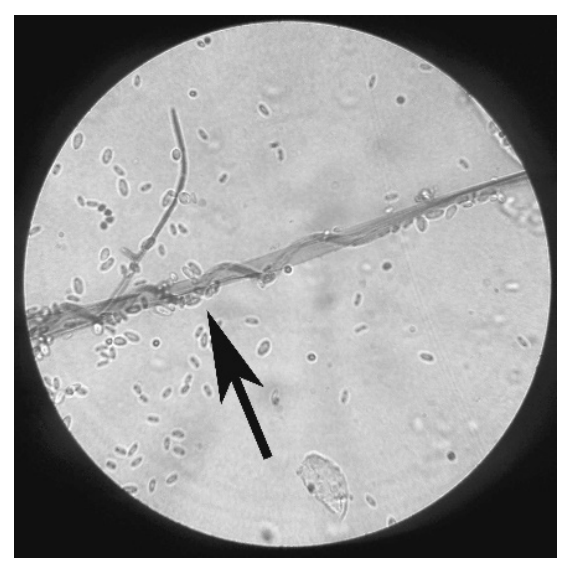

Fig. 5. Microfotografía de la interacción de Trichoderma frente a A. alternata (aumento 1000X). La flecha indica el mecanismo de enrollamiento de las hifas de Trichoderma sobre las hifas del fitopatógeno.

Fig. 5. Photomicrograph of the interaction of Trichoderma against A. alternata (1000X magnification). The arrow indicates the coiling mechanism of the Trichoderma hyphae on the phytopathogen hyphae. 
paranaense, la cual guarda un gran potencial para múltiples aplicaciones biotecnológicas con microorganismos (Gil, 2007; Bich et al., 2021).

Por otro lado, con respecto a los mecanismos de parasitismo observados microscópicamente en los enfrentamientos de Trichoderma frente a A. alternata se pudo observar el mecanismo de "enrollamiento de hifas" y posterior muerte de las hifas del hongo fitopatógeno (Fig. 5).

\section{CONCLUSIONES}

La ejecución del presente trabajo permitió determinar nuevas variantes de cultivo dual para evaluar la capacidad antagónica biocontroladora en condiciones in vitro, sin diferencias significativas a la técnica de cultivo dual tradicional.

Se logró identificar morfológica y molecularmente un aislamiento fúngico fitopatógeno de $A$. alternata, a partir de tejidos vegetales enfermos de yerba mate. Además, se pudo verificar la capacidad fitopatógena de este aislamiento fúngico en tejidos vegetales de yerba mate mediante pruebas de patogenicidad.

Se determinó que 20 de los 30 aislamientos de Trichoderma ensayados presentan gran potencial biocontrolador sobre el aislamiento fitopatógeno obtenido (T. koningiopsis NAN11, T. pleroticola NAN12, T. harzianum POS1, T. atroviride POS2, $T$. longibrachiatum POS5, T. longibrachiatum POS6, T. koningiopsis POS7, T. longibrachiatum POS10, T. stilbohypoxyli POS11, T. harzianum PROF2, T. harzianum PROF3, T. koningiopsis PROF4, T. koningiopsis PROF5, T. harzianum PROF6, T. harzianum PROF7, T. koningiopsis PROF8, T. harzianum TEYU14, T. harzianum TN3, T. citrinoviride TN5 y T. koningiopsis TrichoH). Particularmente, estos aislamientos biocontroladores de Trichoderma presentaron un poder biocontrolador in vitro con diferencias significativas respecto al control positivo del ensayo realizado con la cepa obtenida del producto comercial. Estos resultados reafirman la efectividad de utilizar aislamientos biocontroladores ya adaptados a las condiciones edafo-climáticas del ambiente en donde se encuentra el microorganismo fitopatógeno a controlar biológicamente.

\section{RECONOCIMIENTOS}

Se agradece al Consejo Nacional de Investigaciones Científicas y Técnicas (CONICET) de Argentina y al Comité Ejecutivo de Desarrollo e Innovación Tecnológica (CEDIT) de la provincia de Misiones (Argentina) por las becas de postgrado de Castrillo y Bich.

\section{LITERATURA CITADA}

Ab Rahman, S.F.S., E. Singh, C.M. Pieterse, and P.M. Schenk. 2018. Emerging microbial biocontrol strategies for plant pathogens. Plant Science 267:102-111.

Abbas, M.F., M. Rafiq, A.M. Al-Sadi, S. Alfarraj, S.A. Alharbi, M. Arif, and M.J. Ansari. 2021. Molecular characterization of leaf spot caused by Alternaria alternata on buttonwood (Conocarpus erectus L.) and determination of pathogenicity by a novel disease rating scale. Plos one 16(5):e0251471.

Aloi, F., M. Riolo, S.M. Sanzani, A. Mincuzzi, A. Ippolito, I. Siciliano, A. Pane, M.L. Gullino, and S.O. Cacciola. 2021. Characterization of Alternaria Species Associated with Heart Rot of Pomegranate Fruit. Journal of Fungi 7(3):172.

Alonso, J., y C. Desmarchelier. 2005. Plantas medicinales autóctonas de la Argentina. Bases científicas para su aplicación en atención primaria de la salud. p. 614-617. 1st ed. LOLA (Literature of Latin America), Buenos Aires, Argentina.

Andersen, B., E. Krøger, and R.G. Roberts. 2001. Chemical and morphological segregation of Alternaria alternata, A. gaisen and A. longipes. Mycological Research 105(3):291-299.

Andrade-Hoyos, P., H.V. Silva-Rojas, and O. Romero-Arenas. 2020. Endophytic Trichoderma species isolated from Persea americana and Cinnamomum verum roots reduce symptoms caused by Phytophthora cinnamomi in avocado. Plants 9(9):1220.

Auer, C., and A. Grigoletti. 2002. Association of Fungi with Ilex spp. Boletim de Pesquisa Florestal 45:109-124.

Barnett, I., and B. Hunter. 1998. Illustrated Genera of Imperfect Fungi. 4 ed. Macmillan Publishing Company, New York, USA. p. 240.

Bell, D., H. Wells, and C. Markham. 1982. In vitro antagonism of Trichoderma species against six fungal plant pathogens. Phytopathology 72(4):379-382.

Benítez, T., A. Rincón, M. Limón, and A. Codón. 2004. Biocontrol mechanisms of Trichoderma strains. International Microbiology 7:249260.

Bich G.A., M.L. Castrillo, L.L. Villalba, and P.D. Zapata. 2017. Development of a standard protocol for DNA isolation from leaf-cutting ants suitable for phylogenetic studies and molecular identification. Bulletin of Trends in Biological Sciences 1:27-30. 
Bich, G.A., M.L. Castrillo, F.L. Kramer, L.L. Villalba, and P.D. Zapata. 2021. Morphological and molecular identification of entomopathogenic fungi from agricultural and forestry crops. Floresta e Ambiente 28(2):e20180086.

Burgess, D., and G. Hepworth. 1996. Biocontrol of sclerotinia stem rot (Sclerotinia minor) in sunflower by seed treatment with Gliocladium virens. Plant Pathology 45(3):583-592.

Burris, K.P., F.M. Harte, P.M. Davidson, C.N.Stewart, and S. Zivanovic. 2012. Composition and bioactive properties of yerba mate (Ilex paraguariensis A. St.-Hil.): A review. Chilean Journal of Agricultural Research 72(2):268.274.

Burtnik, O.J. 2006. Yerba Mate: Manual de Producción. Instituto Nacional de Tecnología Agropecuaria (INTA). Agencia de Extensión Rural. Santo Tomé, Corrientes, Argentina. Instituto Nacional de la Yerba Mate (INYM). $52 \mathrm{p}$.

Calistru, C., M. McLean, y P. Berjak. 1997. In vitro studies on the potential for biological control of Aspergillus flavus and Fusarium moniliforme by Trichoderma species. Mycopathologia 137(2):115-124.

Canassa, F., C.P. D'Alessandro, S.B. Sousa, C.G. Demétrio, N.V. Meyling, I. Klingen, and J.I. Delalibera. 2020. Fungal isolate and crop cultivar influence the beneficial effects of root inoculation with entomopathogenic fungi in strawberry. Pest management science 76(4):1472-1482.

Castrillo, M.L., G.A. Bich, P.D. Zapata, and L.L. Villalba. 2016. Biocontrol of Leucoagaricus gongylophorus of leaf-cutting ants with the mycoparasitic agent Trichoderma koningiopsis. Mycosphere 7(6):810-819.

Chaverri, P., F. Branco-Rocha, W. Jaklitsch, R. Gazis, T. Degenkolb, and G.J. Samuels. Systematics of the Trichoderma harzianum species complex and the re-identification of commercial biocontrol strains. 2015. Mycologia 107:558-590.

Choudhary, A.K., N. Singh, and D. Singh. 2021. Evaluation of the bioformulation of potent native strains of Trichoderma spp. against the foot rot/gummosis of Kinnow mandarin. Egyptian Journal of Biological Pest Control 31(1):1-11.

Croge, C.P., F.L. Cuquel, and P.T.M. Pintro. 2021. Yerba mate: cultivation systems, processing and chemical composition. A review. Scientia Agricola 78(5):e20190259.
Cúndom, M.A., y M.G. Cabrera. 2002. Nota corta: Tizón foliar de Celosia argéntea en el nordeste de Argentina. Investigación Agraria: Producción y Protección Vegetal 17(3):463466.

Da Silva, J., N. Suassuna, and W. Bettiol. 2017. Management of Ramularia leaf spot on cotton using integrated control with genotypes, a fungicide and Trichoderma asperellum. Crop Protection 94: 28-32.

Debat, H.J., M. Grabiele, P.M. Aguilera, R.E. Bubillo, M.B. Otegui, D.A. Ducasse, P.D. Zapata, and D. Marti. 2014. Exploring the genes of yerba mate (Ilex paraguariensis A. St.-Hil.) by NGS and de novo transcriptome assembly. PLoS ONE 9(10):e109835.

Druzhinina, I.S., V. Seidl-Seiboth, A. HerreraEstrella, B.A. Horwitz, C.M. Kenerley, E. Monte, P.K. Mukherjee, S. Zeilinger, I.V. Grigoriev, and C.P. Kubicek. 2011. Trichoderma: the genomics of opportunistic success. Nature Reviews Microbiology 9(10):749.

Ferreira, F.V., A.M. Herrmann-Andrade, C.D. Calabrese, F. Bello, D. Vazquez, and M.A. Musumeci. 2020. Effectiveness of Trichoderma strains isolated from the rhizosphere of citrus tree to control Alternaria alternata, Colletotrichum gloeosporioides and Penicillium digitatum A21 resistant to pyrimethanil in post-harvest oranges (Citrus sinensis L. (Osbeck)). Journal of Applied Microbiology 129(3):712-727.

Fontana, D.C., S. de Paula, A.G. Torres, V.H.M. de Souza, S.F. Pascholati, D. Schmidt, and D. Dourado Neto. 2021. Endophytic Fungi: Biological Control and Induced Resistance to Phytopathogens and Abiotic Stresses. Pathogens 10(5):570.

Fraire-Cordero, M.L., D. Nieto-Angel, E. Cárdenas-Soriano, G. Gutiérrez-Alonso, R. Bujanos-Muñiz, y H. Vaquera-Huerta. 2010. Alternaria tenuissima, A. alternata y Fusarium oxysporum hongos causantes de la pudrición del florete de brócoli. Revista Mexicana de Fitopatología 28(1):25-33.

Gauna, M.P., S.A. Gutiérrez, G.A. Bich, M.L. Castrillo, P.D. Zapata, and M.G. Cabrera. 2020. Alternaria alternata causing foliar blight on Chrysanthemum morifolium in Corrientes, Argentina. Summa Phytopathologica 46(1):56-57.

Gil, G. 2007. Misiones: Características físicas y vegetación. En Massoia, Chebez, Bosso (eds.). Los mamíferos silvestres de la provincia de Misiones, Argentina. Editorial L.O.L.A. Literature of Latin America, Buenos Aires, Argentina. 
Guo, Y., A. Ghirardo, B. Weber, J.P. Schnitzler, J.P. Benz, and M. Rosenkranz. 2019. Trichoderma species differ in their volatile profiles and in antagonism toward ectomycorrhiza Laccaria bicolor. Frontiers in microbiology 10:891.

Harman, G., C. Howell, A. Viterbo, I. Chet, and M. Lorito. 2004. Trichoderma species opportunistic, avirulent plant symbionts. Nature Reviews-Microbiology 2(1):43-56.

Hermosa, M., I. Grondona, I. Iturriaga, J. Díaz, C. Castro, E. Monte, and I. Garcia-Acha. 2000. Molecular characterization and identification of biocontrol isolates of Trichoderma spp. Applied and Environmental Microbiology 66(5):1890-1898.

Hernández-Melchor, D.J., R. Ferrera-Cerrato, y A. Alarcón. 2019. Trichoderma: Importancia agrícola, biotecnológica, y sistemas de fermentación para producir biomasa $\mathrm{y}$ enzimas de interés industrial. Chilean Journal of Agriculture \& Animal Sciences 35(1):98-112.

Hosainzadegan, H., R. Khalilov, and P. Gholizadeh. 2020. The necessity to revise Koch's postulates and its application to infectious and non-infectious diseases: a mini-review. European Journal of Clinical Microbiology \& Infectious Diseases 39:215218.

Infante, D., B. Martinez, N. González, y Y. Reyes. 2009. Mecanismos de acción de Trichoderma frente a hongos fitopatógenos. Revista de Protección Vegetal 24(1):14-21.

INYM. 2019. Relevamiento Aerofotogramétrico de Plantaciones. Instituto Nacional de la Yerba Mate (INYM). Disponible en https:// inym.org.ar/noticias/78523-el-relevamientoaerofotogrametrico-de-plantacionesencargado-por-el-inym.html (Consultado: 24 de Marzo de 2021)

Laczeski, M.E., A.L. Onetto, I.J. Cortese, G.Y. Mallozzi, M.L. Castrillo, G.A. Bich, F. Gortari, P.D. Zapata, and M.B. Otegui. 2020. Isolation and selection of endophytic spore-forming bacteria with plant growth promoting properties isolated from Ilex paraguariensis St. Hil. (yerba mate). Anais da Academia Brasileira de Ciências 92:e20181381.

Lezcano, J.C., B. Martínez, y O. Alonso. 2009. Caracterización cultural y morfológica e identificación de 12 aislamientos fungosos de semillas de Leucaena leucocephala cv. Perú. Pastos y Forrajes 32(3):1-14.
Marraschi, R., A.B.M. Ferreira, R.N. da Silva Bueno, J.A.B.P. Leite, C.M.M. Lucon, R. Harakava, L. Garrigós Leite, C.R. Padovani and C.J. Bueno. 2018. A protocol for selection of Trichoderma spp. to protect grapevine pruning wounds against Lasiodiplodia theobromae. Brazilian Journal of Microbiology 50:213-221.

Moya, P., V. Barrera, J. Cipollone, C. Bedoya, L. Kohan, A. Toledo, and M. Sisterna. 2020. New isolates of Trichoderma as biocontrol and plant growth-promoting agent in the pathosystem Pyrenophora teres-arley in Argentina. Biological Control 141:104152.

Ommati, F., and M. Zaker. 2012. Evaluation of some Trichoderma isolates for biological control of potato wilt disease (Fusarium oxysporum) under laboratory and greenhouse conditions. Journal of Crop Protection 1(4):279-286.

Perelló, A., M. Aulicino, C. Martinelli, M. Regueira, V. Moreno, y S. Stenglein. 2015. Caracterización morfocultural de nuevos grupos taxonómicos de Alternaria asociados a enfermedades del trigo en Argentina. Ciencias Morfológicas 17(1):1-15.

Pérez, M.L., M.M. Collavino, P.A. Sansberro, L.A. Mroginski, and E. Galdeano. 2016. Diversity of endophytic fungal and bacterial communities in Ilex paraguariensis grown under field conditions. World Journal of Microbiology and Biotechnology 32:1-15.

Persoon, CH. 1794. Neuer Versuch einer systematischen Eintheilung der Schwämme. Neues Magazin für die Botanik in ihrem ganzen Umfange 1: 63-128.

Piontelli Laforet, E. 2017. Manual de microhongos filamentosos comunes. I.. Viña del Mar, Chile.

Raza, A., A. Razzaq, S.S. Mehmood, X. Zou, X. Zhang, Y. Lv, and J. Xu. 2019. Impact of Climate Change on Crops Adaptation and Strategies to Tackle Its Outcome: A Review. Plants (Basel, Switzerland) 8(2):34.

Sadañoski, M.A., J. Gutierrez-Brower, M.L. Castrillo, A.C. López, P.A. Ojeda, P.D. Zapata, L.L. Villalba, y M.B. Otegui. 2018. Capacidades antagónicas de cepas Trichoderma y su multiplicación en masa usando desechos agrícolas. Revista de Ciencia y Tecnología 30(1):4-11.

Salvatierra-Martínez, R., G. Sepúlveda-Chavera, W. Huanca-Mamani, and M. RodríguezMolina. 2015. Strains of Trichoderma from Northern Chile: Adaptive tolerance in boric saline soils. Interciencia 40(4):263-269. 
Schoch, C.L., K.A. Seifert, S. Huhndorf, V. Robert, J.L. Spouge, C.A. Levesque, W. Chen, and Fungal Barcoding Consortium. 2012. Nuclear ribosomal internal transcribed spacer (ITS) region as a universal DNA barcode marker for Fungi. Proceedings of the National Academy of Sciences 109:6241-6246.

Toghueo, R.M., P. Eke, I.Z. González, B.R. de Aldana, L.W. Nana, and F.F. Boyom. 2016. Biocontrol and growth enhancement potential of two endophytic Trichoderma spp. from Terminalia catappa against the causative agent of common bean root rot (Fusarium solani). Biological Control 96:8-20.
White, T.J., T. Bruns, S.J.W.T. Lee, and J.W. Taylor. 1990. Amplification and direct sequencing of fungal ribosomal RNA genes for phylogenetics. PCR protocols: a guide to methods and applications 18:315-322. 